\title{
Study on the Design of Greenhouse Monitoring System based on Zigbee Wireless Communication Technology
}

\author{
Jie Huang ${ }^{1, a}$ \\ ${ }^{1}$ College of Mechanical and Electronic Engineering, Hezhou University, Hezhou, China, 542899 \\ ${ }^{a}$ email
}

Keywords: Greenhouse Monitoring System, Zigbee Technology, Wireless Communication

\begin{abstract}
With intelligent greenhouse computer, network, electronic, automatic control, and other high-tech means to achieve automatic detection and control of plant growth small environment temperature, humidity, light, soil moisture, ventilation and other environmental parameters, breaking the traditional agricultural production is subject to weather , regional, seasonal and other factors limit the year to provide the best possible environment for the growth of different plant nutrition and moisture, shorten the growth cycle of crops, improve crop yields and quality. Intelligent Greenhouse become modern agricultural production techniques typical representative of the world today is the development direction of farming production.
\end{abstract}

\section{Introduction}

Wireless sensor networks with other wired network does not have some of the features: the ability to set up large-scale networks, the traditional network nodes typically only a few hundred, and wireless sensor networks can be constructed hundreds of thousands or even tens of thousands of nodes in sensor detection system ; wireless sensor network self-organization functions, some nodes may work process fails, or electricity and other reasons lost contact with the network, some nodes will be necessary to move the position, or to add new nodes at certain locations, the network can dynamically manage and configure the nodes can be dynamically adjusted according to changes in the network structure of network nodes; wireless sensor networks to detect a data-centric wireless networks, users mainly focus on the data sent or received, and less concerned about the position of the sensor node information.

ZigBee technology is based on IEEE802.15.4 standard wireless communication technologies in 2005, ZigBee Alliance released the ZigBee specification V1.0, IEEE802.15.4-2003 defines the physical layer PHY and MAC standard media control layer and network layer on the support services application. Then on the ZigBee Alliance ZigBee standard upgrade ZigBee2006 launched in 2006. We launched ZigBee2007 / PRO protocol specification in 2007.

1) network capacity to accommodate multiple node ZigBee network can accommodate up to 65,500 wireless sensor nodes, can meet the requirements of medium and small size of the various monitoring systems. 2) Power low power consumption, save energy ZigBee node at work only $1 \mathrm{~mW}$, the general use of intermittent work, most of the time in a dormant state, the node power consumption is very small, two batteries can work for about a year. 3) Due to low power short distance communication between ZigBee node communication distance is usually a few meters to tens of meters, if the need for further data communication node circuit design, to add RF power amplifier module. 4) Safety performance ZigBee provides CRC data validation, data transmission using AES-128 encryption algorithm, has higher data transmission security. 5) Data transmission rate is low ZigBee working frequency of use of $2.4 \mathrm{GHz}$, its data transfer rate of $250 \mathrm{Kbps}$, due to the wireless sensor applications typically do not need to transfer large amounts of data, so the data transfer rate can meet the vast majority of sensor networks demand. 6) Low cost for a wide range with the sharp increase in usage market, the price ZigBee chips and wireless node modules continued to decline, to meet the demand for more cost-sensitive applications. Additionally, ZigBee protocol does not receive royalties, is also an important factor in its cost reduction. 


\section{System Architecture Design}

In order to fully detect the temperature inside the greenhouse in different regions, humidity, light and other environmental parameters, you need a lot of different functions arranged sensor for detecting changes in environmental parameters of the regional situation, the implementation by the master computer control equipment, interior small greenhouse environment automatically adjusted to achieve the best environment for the growth of crops needed. In order to meet the actual needs of different size greenhouse, and enhance system flexibility, versatility, by several hardware solutions comparative demonstration greenhouse environment monitoring system decided to adopt a tree topology.

In order to ensure long-term reliable operation of the system, the network coordinator using the AC power supply. Implement monitoring functions mainly by the host PC, in addition, it can also be used to view the various functions and settings configured by the network coordinator keyboard display. Total hubs as the network coordinator to monitor the host and other nodes in the exchange of information, on the one hand via RS232 RS485 serial bus or with the host monitoring host, on the one hand to exchange data with the routing node or wireless sensor nodes. System operation, the network coordinator receives environmental parameters detected by the sensor is then sent to the host, the host will send control commands sent to the routing node, and real-time detection and display of network status. Monitoring host In addition to managing wireless sensor networks, but also the docking operation processing environmental parameters received, then the control mechanism for heating, humidification, ventilation, shading, gassing, watering, fertilizing, etc., to achieve unmanned automatic greenhouse environment control, to create the best environment suitable for crop growth.

Master node router module is mainly composed of CC2430 chip configuration, CC2591 RF front-end power amplifier module, antenna, debug interface, power modules, keyboards, monitors and other components. Relay router node is mainly used for data between the coordinator and the sensor nodes forwarding and routing tables brought home maintenance, according to the actual needs of the sensor module can also be configured to collect environmental information. CC2591 RF front-end power amplifier module is optional, when a large greenhouse area, plus CC2591 module away from the router node when the sensor node can increase the signal transmission distance, building large ZigBee wireless network. However, the power amplifier module is also a corresponding increase in power consumption of the device, if the battery-powered, reliable may cause reduction in working hours.

Wireless sensor nodes, the network terminal node, the main module of the CC2430 chip configuration, the temperature sensor and interface circuit, a humidity sensor and interface circuit, antenna, debug interface, power modules, keyboards, monitors and other components. Sensor nodes are monitored channel forefront, temperature and humidity data collection points for the conservatory, as the main basis for environmental control equipment for automatic control. To reduce power consumption, using the LCD display shows the scene of the sensor temperature and humidity data, to facilitate staff to view. To facilitate mobile node, the sensor node is battery powered mode. Another configuration of the solar cell power supply module, if the sensor device operating current, can be easily powered by solar cells, without laying additional power lines.

\section{System Hardware Module Design}

ZigBee main module is the core component of the network coordinator node and router nodes and wireless sensor nodes share due CC2430 is a highly integrated chip integration of RF, MCU, memory, ADC, USART, DMA and other features of the system, therefore the main module only few external components, could constitute meet master node function module, all functions required by the system. CC2430 ZigBee module consists of the main chip, reset circuit, filter circuit, clock circuit, PCB antenna and other components, in order to achieve the desired performance, the company strictly in accordance with TI's reference circuit for circuit design. In order to increase the signal transmission distance, nodes can also be used whip antenna or chip antenna, a whip antenna 
belonging unbalanced single-ended antenna, standard SubMiniatureVersionA (SMA) antenna connector, with good performance and signal transmission distance, high sensitivity, but there are volume, high price disadvantage, particularly should a reasonable choice based on the actual situation of greenhouse monitoring and control of the scene. When the distance between wireless nodes farther, decreased reliability of signal transmission, the circuit node should be added to the core of the long-distance CC2591 RF front-end power amplifier module to ensure reliable transmission of wireless signals.

When building large greenhouse monitoring system, routing nodes and distant from the sensor nodes, resulting in unstable signal transmission system reliability decline. TI company to address this issue specifically introduced excellent performance 2.4-GHzRF front-end power amplifier chip CC2591. CC2591 integrates a high-performance power amplifier PA, a low-noise amplifier LNA, RF matching network, balun and other components. Fewer external components required to interface circuit CC2591, CC2430 and the interface is simple, easy to use. After the node joins the CC2591 power amplifier circuit, significantly increase the signal transmission distance, but it will also increase the operating current. Therefore, in the case of battery-powered nodes, should be used in a large-capacity high-quality batteries, the case of conditions can also be used external power or AC power.

RS-232C is the American Electronics Industry Association (EIA) to formulate and release serial communication interface standard, originally developed for the use of public telephone networks and modems for data communication distance. RS-232C serial interface and the computer has now become the most widely used industrial control serial communication interface standards. In order to increase the transmission distance of serial data, improve the signal anti-jamming capability, RS-232C standard specifies a dedicated RS-232C signal levels, with negative logic and bipolar signals indicates the level of data transmission.

RS232C interface transmitting terminal: $+5 \mathrm{~V} \sim+15 \mathrm{~V}$ represents a logic 0 (or called SPACE empty, corresponding to the ON state), $-5 \mathrm{~V} \sim-15 \mathrm{~V}$ indicates a logic 1 (or called MARK mark, to be applied to the OFF state). The actual voltage using a different computer or device is not the same, as long as the voltage value will be able to communicate properly within the specified range. RS232C interface receiving terminal: To reduce the impact of noise voltage, the receiver has increased the range of high and low voltage, a predetermined $+3 \mathrm{~V} \sim+15 \mathrm{~V}$ voltage is logic $0,-3 \mathrm{~V}$ $\sim-15 \mathrm{~V}$ to logic 1 . If the received signal voltage between $-3 \mathrm{~V} \sim+3 \mathrm{~V}$, serial interface can not correctly identify, it is possible to send high-level computer, may send low. RS232C transmitter and receiver common ground, the signal levels are sent and received data to the common ground as a reference, this transmission is called unbalanced transmission mode. When the signal is transmitted common-mode noise voltage superimposed voltage signal to the receiving end, the receiving data error. So RS-232C standard is represented by a high voltage and a wide range of high and low voltage, to reduce the impact of noise voltage.

RS-232C data transmission rate and transmission distance are relatively small. RS-232C standard specifies a maximum data transfer rate of 20Kbps. When the baud rate is $19.2 \mathrm{Kbps}$, the maximum transmission distance is $15 \mathrm{~m}$. Practical application, by reducing the baud rate and the use of shielded wire and other measures to increase the transmission distance.

\section{Software Design}

Temperature and humidity sensor acquisition program design. When the MCU sends commands to SHT 11, first by starting the transfer process initialization, and then issue the command, the command contains five command bits and 3000 address bits. When the MCU control SHT11 temperature and humidity measurements, temperature measurement by issuing the command 00000011B start temperature A / D conversion process by issuing the command 00000101B start measuring humidity humidity A / D conversion process, select different resolutions, different time required for conversion , 8-bit resolution conversion time of about 20ms, 12-bit resolution conversion time of $80 \mathrm{~ms}$, 14-bit resolution conversion time of 320ms. When the A / D conversion, SHT 11 the data line low, MCU can detect the data line status inquiry conversion is 
complete. Due to the high-resolution conversion time will be extended, so select the resolution should be based on the specific application requirements.

ZigBee node software design. ZigBee wireless node is divided into software design and application programming node ZigBee protocol stack two parts. The role of the node application is to achieve complete node specific functions, ZigBee protocol stack for communication and data transmission ZigBee network. Wireless sensor node to the environmental data collected through a router node network coordinator, the coordinator via RS232 or RS485 bus to the monitoring host, the data from the host computing, display, processing and compared with the set value, the control mechanism implementation to achieve automatic control of environmental parameters. Host monitoring and management software use VB6.0 language and ACCESS database development, VB6.0 serial port for data exchange with the network coordinator through the MSComm control.

The main function of monitoring software are: 1) to receive each network coordinator sent the temperature and humidity data, according to the preset value, comprehensive data analysis and processing, send control commands to control operation of the equipment to the controller. 2) the timing of environmental data to the database storage temperature and humidity. 3) control panel interface display control equipment operating status and fault information tips, equipment manual, automatic control switch. 4) the master interface real-time display of the monitoring points within the greenhouse temperature and humidity data, in order to facilitate visualize temperature changes, when the measured temperature exceeds the set limit value, the red alarm, when the measured value exceeds the limit, the blue alarm prompt, when the measured value within the set limits, the temperature display window in green.

\section{Conclusion}

Our greenhouse monitoring system mostly uses traditional wired communication. Due to the need to lay a large number of signal transmission lines and power lines, installation of long construction period, high cost, the line easy to aging, poor reliability, increase the update, maintenance complexity, reduces the reliability and flexibility of space monitoring, giving the greenhouse monitoring extension systems brought obstruction. In this paper, the existence of deficiencies in the current system to monitor greenhouse, which lasted six months, the successful completion of the design of intelligent greenhouse environment monitoring system based on ZigBee wireless network, through laboratory simulation run, the user greenhouse field operation, the performance indicators have reached the target to improve the degree of automation of agricultural production, saving production costs, create higher economic returns.

\section{References}

[1] ZigBee and Bluetooth-Competitive or Complementary. Venkat Bahl, 2002.9.

[2] DS18B20 Programmable Resolution 1-Wire Digital Thermonmeter.www.maxim-ic.com

[3] Audun Andersen. Implementation of Microstrip Balun for CC2420 and CC243x.

[4] Shizhuang Lin, Jingyu Liu, Yanjun Fang. ZigBee Based Wireless Sensor Networks and Its Applications in Industrial [J]. Proceedings of the IEEE International Conference on Automation and Logistics2007, 8, 18-21.

[5] IEEE 802.15.4 Group. Wireless medium access control (MAC) and physical layer (PHY) specifications for low rate wireless personal area networks (LR WPAN) [J]. IEEE 802. 15.4 Group, 2006. 